\title{
Notice to the Authors and Readers of Neuropediatrics - Online Manuscript Submission in Preparation
}

\section{Dear Readers and Authors,}

Our international journal has reported research results in its field for over 35 years. During this time the quality and strength of the journal in the field has grown considerably. As a reader of Neuropediatrics you will already be aware of the excellence and relevance of the information provided in this publication. In 2004 our ISI impact factor increased yet again up to $\mathbf{1 . 6 1 9}$ and interest and support in the journal has been unwavering.

An additional development for the journal has been the introduction of eFirst, the platform for advanced online publication. Approved papers appear online several weeks before they are published in print providing faster access to the most recent and up-to-date research publications.

In order to facilitate the whole reviewing process, the publisher has now decided to provide an online submission system for both authors and editors of Neuropediatrics. The system will support the complete process of submitting and reviewing manuscripts, as well as the correspondence between authors, editorial office and the reviewers. The system will be launched on November 1st, 2005. Until then, manuscript submissions shall be executed in the traditional way.
Important: Beginning from November 1st, 2005, authors are asked to refrain from submitting paper copies of their manuscripts and to exclusively use the online submission system. Please note that after November 1st no paper copies will be accepted at all.

The URL address of the system will be disclosed in the October issue of Neuropediatrics. In addition to this, authors are asked to follow the information given on the journal's home page at www.thieme.de/neuropediatrics. As soon as the submission system is online, the publisher will place a hyperlink at the journal's page.

The publisher and editors of Neuropediatrics are confident that the implementation of the online submission system will represent an important improvement for our authors. For this purpose, Thieme is cooperating with the world's leading provider of manuscript tracking systems. The system itself will help to reduce the administrative time spans and thus shorten the publication time. It is easy to operate and offers a maximum of convenience to authors and editors.

The publishers will provide any help needed for the time of transition to the new system, and we kindly ask the authors to support the work of the Editorial Office by using the new facilities.

The publishers and the editors 